\title{
Dysgraphia: evaluating an Arabic training program for remediation of Egyptian dysgraphic children
}

\author{
Mohamed E. Darweesh ${ }^{1 *}$, Safaa R. Elsady², Nahla A. Reifaie ${ }^{2}$ and Rania M. Sidhom
}

\begin{abstract}
Background: Dysgrahia is a learning disability that affects writing; it requires a complex set of motor and information processing skills. Dysgraphia makes the act of writing difficult and leads to problems with spelling, poor handwriting, and putting thoughts on paper. People with dysgraphia can have trouble organizing letters, numbers, and words on a line or page. The purpose of the present study was to evaluate an Arabic training program for remediating Egyptian Arabic-speaking dysgraphic children.

Results: The Arabic training program through a quasi-experimental study (pre-post-test) showed significant results in remediating dysgraphia on twenty cases that had attended the phoniatric unit complaining of learning disability having dysgraphia with or without dyslexia.

Conclusion: The Arabic dysgraphia training program is an effective multisensory approach tool in the training of different types of dysgraphia.
\end{abstract}

Keywords: Dysgraphia, Learning disabilities, Multisensory program

\section{Background}

Dysgraphia makes the act of writing difficult and leads to problems with spelling, poor handwriting, and putting thoughts on paper. People with dysgraphia can have trouble organizing letters, numbers, and words on a line or page. Dysgraphia can be a language-based and/or non-language-based disorder [1]. Many people have poor handwriting, but dysgraphia is more serious.

Dysgraphia is a neurological disorder that generally appears when children are first learning to write. Experts are not sure what causes it, but early treatment can help prevent or reduce problems. Writing requires a complex set of motor and information processing skills. Not only does it require the ability to organize and express ideas in the mind, but it also requires the ability to get the

\footnotetext{
* Correspondence: mohameddarwish@hotmail.com

${ }^{1}$ Unit of Phoniatrics, ENT Department, Faculty of Medicine, Tanta University,

Tanta, Egypt

Full list of author information is available at the end of the article
}

muscles in the hands and fingers to form those ideas, letter by letter, on paper [2].

Even with the increased use of computers and tablets, handwriting remains an important skill that school-aged children have to master, because the motor action of creating letters on paper has been found to increase the memory of letters beyond that attainable with keyboarding alone. James [3] found that the creation of letterforms augmented the visual processing of letters in preschool children. Thus, the importance of learning to manually form or draw letters cannot be underestimated [4]. There are different international tools for the remediation of dysgraphia; however, a specific program for Arabic Egyptian children was not available and that was the main purpose of this study to design and evaluate a program for remediation of Arabic Egyptian dysgraphia.

The Arabic script is a writing system used for writing Arabic and several other languages of Asia and Africa, such as Persian, Kurdish, Sindhi, Balochi, Pashto, Lurish, Urdu, and Kashmiri [5]

\section{Springer Open}

(c) The Author(s). 2020 Open Access This article is licensed under a Creative Commons Attribution 4.0 International License, which permits use, sharing, adaptation, distribution and reproduction in any medium or format, as long as you give appropriate credit to the original author(s) and the source, provide a link to the Creative Commons licence, and indicate if changes were made. The images or other third party material in this article are included in the article's Creative Commons licence, unless indicated otherwise in a credit line to the material. If material is not included in the article's Creative Commons licence and your intended use is not permitted by statutory regulation or exceeds the permitted use, you will need to obtain permission directly from the copyright holder. To view a copy of this licence, visit http://creativecommons.org/licenses/by/4.0/. 
The Arabic script is written from right to left in a cursive style, in which most of the letters are written in slightly different forms according to whether stand alone or are joined to a following or preceding letter. The basic letter form remains unchanged also it does not have capital letters [6].

Arabic calligraphy is the artistic practice of handwriting and calligraphy based on the Arabic alphabet. It is known in Arabic as khatt, derived from the word "line," "design," or "construction" [7].

Naskh and ruq ah calligraphy are the most popular taught at Egyptian schools.

\section{Types of dysgraphia [8]}

(I) In dyslexic dysgraphia, the spontaneously written text is illegible, especially when the text is complex. Oral spelling is poor, but drawing and copying of written text are relatively normal. Finger tapping speed (a measure of fine motor speed) is normal [9]. (II) Motor dysgraphia is due to deficient fine motor skills, poor dexterity, poor muscle tone, or unspecified motor clumsiness. Letter formation may be acceptable in very short samples of writing, but this requires extreme effort and an unreasonable amount of time to accomplish, and it cannot be sustained for a significant length of time. Overall, their written work is poor to illegible even if copied by sight from another document, and drawing is difficult. Oral spelling for these individuals is normal, and their finger tapping speed is below normal. This shows that there are problems within the fine motor skills of these individuals. Writing is often slanted due to holding a pen or pencil incorrectly. The components of motor dysgraphia are sometimes related to anatomical problems, executive dysfunction, and motor planning deficits [9]. (III) In spatial dysgraphia, Children with spatial dysgraphia have a defect in the understanding of space and display illegible writing whether spontaneously produced or copied. Oral spelling and finger tapping speed are normal, but drawing is very problematic [10].

The aim of this study was to design and evaluate an Arabic training program for remediating Arabicspeaking dysgraphic children, to be used as an adjuvant part of the therapy program for learning disabilities.

\section{Methods}

\section{Subjects}

A quasi-experimental study (pre-post-test) was applied on twenty cases 14 males and 6 females that had attended the phoniatric unit complaining of learning disability having dysgraphia with or without dyslexia that was chosen randomly after fulfilling the inclusion criteria. This is an uncontrolled study that means all the participants are given a treatment and simply followed for a period of time to see if they improve, with no comparison against another group (control group) that is either taking another treatment or no treatment at all.

Children participated in the study were selected according to the following criteria:

1. Inclusion criteria

(a) Age group between 6.5 and 10 years old

(b) IQ $\geq 90$

(c) Dysgraphic children, according to the assessment protocol

2. Exclusion criteria

(a) IQ $>90$

(b) Associated sensory deficit (hearing or visual disorders)

\section{Methods}

Assessment protocol

Every case was pre-diagnosed by the following protocol of assessment:

1. Elementary diagnostic procedures

(a) History taking

(b) General examination

(c) Vocal tract examination

(d) Ear and nose examination

(e) Neurological examination

(f) Auditory perceptual assessment of language and speech

2. Clinical diagnostic aids

(a) Stanford-Binet "4th Arabic version" to provide mental age and IQ [11].

(b) Complete learning disability battery

I Language test by PLS4-modified test [12]

II Illinois test of psycholinguistic abilities [13]

III Phonological awareness test when possible

[12]

IV Dyslexia screening test [14]

V Dysgraphia disability scale [15]

\section{Remediation by the constructed training program}

Material of the therapy was constructed depending on different international sources and from the available material for therapy of dyslexia in the form of sensory, visual, auditory, and kinesthetic materials forming a multisensory program that aims at enhancing the sensory motor development using the sensory integration strategy which is increasing sensory input through meaningful activities leading to improving the ability to process sensory information and enhance learning, i.e., increasing sensory integration will increase the quality of motor output.

Each child was allowed to apply the program twice per week for $40 \mathrm{~min}$ each for 6 month; then, re-evaluating the condition of each child was done blindly without previewing his previous test results after 6 months from 
the beginning of the training was done using the following:

\section{- Dysgraphia Disability Scale [15]}

\section{Data management and analysis}

The collected data was revised, coded, tabulated and introduced to a PC using Statistical package for Social Science (SPSS 16.0 for windows; SPSS Inc., Chicago, IL, 2001). Data was presented and suitable analysis was done according to the type of data obtained for each parameter.

1. Descriptive statistics

(a) Mean

(b) Standard deviation $( \pm \mathrm{SD})$

(c) Frequency and percentage of non-numerical data

2. Analytical statistics

(a) Paired sample $t$ test. A parametric test was used to compare mean scores between pre- and posttraining results regarding the tested items.

(b) Bivariate $r$ correlation. A parametric test used to examine the relationships between selected variables.

3. $P$ value: level of significance

(a) $P>0.05$. Non-significant (NS)

(b) $P<0.05$. Significant $(\mathrm{S})$

\section{Results}

Twenty cases of dysgraphic children were chosen randomly from patients attending the phoniatric unit hospital 14 males and 6 females; 18 of them was dyslexic dysgraphia one spatial and one motor dysgraphia.

The average results of dysgraphic children pre- and post-training are presented in terms of means, standard deviations.

Table 1 shows that patients who had normal handwriting with no disability increased from $0 \%$ pre-training to reach $15 \%$ after training. Moreover, patients who had good handwriting with minimal disability increased from $20 \%$ pre-training to reach $55 \%$ after training. On the other hand, patients had mild to moderate disability decreased from $50 \%$ pre-training to $30 \%$ after training. Those patients who had severe disability (disable) decreased from $30 \%$ pre-training to $0 \%$ after training.

Table 2 shows the age of each patient and his/her score in the dysgraphia disability scale.

Table 3 shows that there was a statistical significant difference in the total mean scores pre-training and post-training where as $t$ test (9.992) with $p$ value (0.000).

As shown in Table 4 , there were statistically significant differences $(p<0.05)$ between pre- and post-training results of each item of the handwriting subtest of the DDS, respecting lines, spacing between words, letter direction, spelling a sentence, and punctuation.

Table 5 shows statistically significant differences $(p<$ 0.05 ) between pre- and post-training results only in the second item which is the ability to pour a glass of water into another one.

\section{Discussion}

After the success of the therapy program applied for learning-disabled "dyslexic" children, it was mandatory to construct a therapy program for dysgraphia as a complementary practice "tool" to complete the tools needed for learning skills [8].

The present study aimed at constructing a therapy program as a remediation of dysgraphia in affected Egyptian Arabic-speaking children aged from 6.5 to 10 years with IQ more than or equal 90 . The research design used was a pre-test-post-test design. The proposed dysgraphia therapy program for the Egyptian Arabic-speaking learning-disabled children aimed to target dyslexic, motor, and spatial dysgraphia as classified by Deuel [8].

The proposed multisensory therapy program items go with the fact that handwriting involves the synthesis of many skills including orthographic coding, finger function, visual motor integration, eye/hand coordination, and visual perception [16]. These skills were found to be successfully managed with appropriate intervention.

Table 1 Frequency percentage distribution of dysgraphia disability scale ( $N, 20$ cases)

\begin{tabular}{lll}
\hline Scoring of DDS & \multicolumn{1}{c}{$\begin{array}{l}\text { Pre-training } \\
\text { No. of cases }\end{array}$} & $\begin{array}{c}\text { Post training } \\
\text { No. of cases }\end{array}$ \\
\hline Normal handwriting with no disability (20-17) & $0,0 \%$ & $3,15 \%$ \\
Good handwriting with minimal disability (16.75-13) & $4,20 \%$ & $11,55 \%$ \\
Mild to moderate disability (fair ability) (12.75-9) & $10,50 \%$ & 6,30 \\
Severe disability (disable) (8.75-5) & $6,30 \%$ & $0,0 \%$ \\
Total disability (4.75-0) & $0,0 \%$ & $0,0 \%$ \\
\hline
\end{tabular}

DDS Dysgraphia Disability Scale 
Table 2 The score of the Dysgraphia Disability Scale for each child pre- and post-training

\begin{tabular}{llll}
\hline Case & Age (years) & Pre-training score & Post-training score \\
\hline 1 & 8.5 & 15.25 & 17 \\
2 & 9 & 12.25 & 16.75 \\
3 & 6.5 & 10.5 & 13 \\
4 & 9.2 & 14 & 17 \\
5 & 8 & 7.75 & 16.75 \\
6 & 7.2 & 8.75 & 15.25 \\
7 & 10 & 13.5 & 16.75 \\
8 & 6.8 & 7 & 10.75 \\
9 & 10 & 10.25 & 16.5 \\
10 & 7.9 & 12 & 14.75 \\
11 & 7.2 & 12.25 & 19.75 \\
12 & 6.8 & 8.75 & 11.75 \\
13 & 8.8 & 8.75 & 12.25 \\
14 & 10 & 11 & 16 \\
15 & 8 & 9.75 & 12.75 \\
16 & 9 & 10.75 & 12 \\
17 & 9 & 12 & 16 \\
18 & 10 & 13.75 & 16.5 \\
19 & 6.6 & 8.75 & 12.25 \\
20 & 6.6 & 11.25 & 14.5 \\
\hline
\end{tabular}

Application of the multisensory therapy program on the dysgraphic children gave rise to the following

\section{observations}

Generally, the significant improvement in various parameters especially the handwriting subtest of the dysgraphia disability scale in the children post-training indicates success of the proposed therapy program which offered scientifically based instructions to the children.

The proposed therapy program provides multisensory activities in the form of visual, auditory, and kinesthetic activities depending on the fact that handwriting is one of the most challenging fine motor tasks a child is required to learn at school. There are many different skills involved as bilateral coordination, hand dominance and strength, visual memory, discrimination, and sequencing. Introducing all of

Table 3 Comparison of total mean scores between pre- and post-training results of dysgraphia disability scale ( $N, 20$ cases) (done by paired sample $t$ test)

\begin{tabular}{lll}
\hline & Pre-training & Post-training \\
\hline$X \pm$ SD & $11.5 \pm 2.46$ & $15.3 \pm 1.94$ \\
$t$ test & 9.992 & \\
$p$ value & 0.000 & \\
\hline
\end{tabular}

$S D$ standard deviation these meaningful activities together will lead to improving the ability to process sensory information and enhance learning, i.e., increasing sensory integration will increase the quality of motor output as stated by Bundy and Murray [17].

\section{Program application}

The activities of the program were arranged in a systemic way from the simple to the more complex.

The program began by orientation to all the letters through singing the alphabet song, reading flash cards having the letters, and a word starting with it as most of the cases were dyslexic and already have problems with reading the letters. Then, the children started to draw geometric shapes that may form a part of the letters to break the ice of writing as most of them are frustrated and afraid of writing tasks then shifted to write the letter inside marker. Writing and tracing the letter over and over again increased the stereo-gnostic experience that imprints the letter formation into the child's brain; the child can practice tracing letters through preprinted letters in the form of dashes or dots or a solid marker line, but we found that it is easy and more successful for the children to trace a solid highlighted line than tracing dashes or dots. Malburg and Coventry [18] reported the same point. Each letter has a word starting with it as an example, so the child was asked to segment it out loud and to color the picture of this word, Coloring inside the borders is a very hard disappointing task for children as it causes hand muscle exhaustion and pain, coloring with a brush is fun for kids and reduces pain in their shoulders then they can shift to pencils as coloring trains the hands to be flexible with holding the pencil and this what was followed in our program. Kumar [19] recommended that the children are advised to rest their hypothenar muscles on the table and keeping middle finger beneath the pencil just for support (this position is the normal position and movement for writing); we followed it in a gentle way in order not to dissociate the child's attention.

Then, the children trace the letter shape and form it with play dough; these activities were extremely fun to the children and very helpful as they recall the letter shape. Winston and Professor [20] stated that activities that improve the muscle strength as molding playdough and tracing pictures and letters lead to improvement in the hand muscles and resulted in a better control of the pen during writing. We used activities as writing in sand, in rice, in air, and on a rough surface with color on the index finger that helped a lot through enhancing the child visual memory to be able to retain the learning required for reading and writing and increase the speed of its production as it also increased the sensory stimulation of the child fingers [21]. 
Table 4 Comparison of total mean scores between the pre-training and post-training results regarding the handwriting subtest in the dysgraphia disability scale ( $N, 20$ cases) (done by paired sample $t$ test)

\begin{tabular}{llll}
\hline Handwriting subtest & Pre, $X \pm$ SD & Post, $X \pm$ SD & $t$ test*, $P$ value \\
\hline 1. Respecting lines & $0.41 \pm 0.34$ & $0.91 \pm 0.46$ & $6.89^{*}, 0.000$ \\
2. Spacing between words & $0.28 \pm 0.30$ & $0.80 \pm 0.20$ & $6.76^{*}, 0.000$ \\
3. Letter direction & $0.35 \pm 0.29$ & $0.83 \pm 0.21$ & $6.43^{*}, 0.000$ \\
4. Spelling a sentence & $0.28 \pm 0.30$ & $0.61 \pm 0.26$ & $5.37^{*}, 0.000$ \\
5. Punctuation & $0.17 \pm 0.34$ & $0.55 \pm 0.32$ & $6.38^{*}, 0.000$ \\
\hline
\end{tabular}

* = significant

SD standard deviation

Modeling the letter by the trainer to the child with explaining its phonetic description by writing large and preferably on a vertical surface or unlined paper while explaining how the letter is drawn, writing large-size letters gave more input to the child so he or she can use gross motor movements to learn the motor patterns. Due to poor fine motor skills and feedback from the hands, these children cannot reliably learn how to form their letters writing in a normal size. These steps were followed according to Smith [21] who stated that writing instruction should start at a chalkboard, white board, or on a strip of butcher paper taped to the wall. As the child learns the letter, instruction can move to smaller spaces on unlined paper then lined paper. A child needs to position the wrist correctly in slight extension (the position your wrist is in when waving). Working in the vertical plane at the chalkboard puts the wrist in the correct position for writing. On the other hand, some children hook their wrist which makes it more difficult to control the pencil and may benefit from using a slant board at the desk.

When moving to writing on paper, we ensured that the child writing position is right. By making the edge of the paper parallel to the child's writing arm. For righthanders, the right corner of the paper higher and lefthanders with the left corner higher than the right. This helped to prevent the hooking motion sometimes adapted by left-hand writers [22].

As soon as the child learned the letters, we allowed him or her to start practicing the letters in short words. The child was encouraged to keep the focus on making the letters right, not to slip into writing quickly and poorly. At this point of the learning, children were advised not to write too quickly, the focus is on learning the letter formations. The child was asked to put a smiley face or star beside the letter that he/she thought looks the best (to practice self-evaluation) [23, 24].

Jordan et al. [25] concluded in their study that $10 \mathrm{~min}$ per day writing exercises improves legibility of hand writing; however, it does not affect the speed of writing.

After finishing all the letters discussing similarities and differences of letters having similar shapes was done so as to be easier to recall as advised by Heerlen [24].

Auditory training included activities as singing the alphabet song, saying the sound of each letter aloud while writing it, hearing and reading flash cards, segmenting words aloud saying the sound and name of each letter aloud, and auditory description for how to write each letter (see enclosed material in the bag). The child was asked to talk aloud through these activities as talking aloud is considered a cognitive intervention which uses self-talk and verbal guidance to improve occupational performance [24].

Verbal prompt was commonly done while the child was writing and it helped a lot. This goes with Lippincott [22] who reported that prompt enhances working memory, being devoted to remembering how to form a letter, not leaving space to focus on ideas and grammar. It is helpful to mark a dot on the starting points on letters that are formed properly.

Mama and Ichtb [26], proved in their study that the production effect (saying words aloud) is a well-known memory phenomenon that refers to the benefit in memory for "read aloud words" which gave better effects relative to words read silently. Although other learning

Table 5 Comparison of total mean scores between the pre-training and post-training results regarding the fine motor function subtest in the dysgraphia disability scale ( $N, 20$ cases) (done by paired sample $t$ test)

\begin{tabular}{llll}
\hline Fine motor function subtest & Pre, $X \pm$ SD & Post, $X \pm$ SD & $t$ test ${ }^{*}, P$ value \\
\hline 1. Grip & $0.83 \pm 0.27$ & $0.92 \pm 0.14$ & $1.92^{*}, 0.69$ \\
2. Pour a glass of water into other & $0.81 \pm 0.21$ & $0.93 \pm 0.15$ & $2.93^{*}, 0.008$ \\
3. Putting a coin in a safe box & $0.92 \pm 0.16$ & $0.96 \pm 0.122$ & $1.37^{*}, 0.186$ \\
4. Buttoning & $0.800 \pm 0.27$ & $0.88 \pm 0.249$ & $1.92^{*}, 0.69$ \\
\hline
\end{tabular}

*= significant

SD standard deviation 
methods have been found to improve memory, it is vocal production that seems the robust and most consistent.

The process proceeded from reading to copying then dictating already seen words or paragraphs then lastly dictating novel words and paragraphs from the Arabic curriculum according to grade of each child to enhance the orthographic visual skills and to be trained on good handwriting and increase legibility.

The children were allowed to do some activities throughout the training as forming beads, playing video games, puzzles, and games to discriminate right from left in order to have sequencing skills to form letters correctly, spell words, and form sentences. Many fine motor activities such as putting beads in the correct order with visual cues at first then without cues enhances visual sequencing memory. Very young children learn sequencing skills as they recite the $\mathrm{ABC}$ song auditory sequencing [22]. Video games enhance visual memory and games that require writing encourage children how to write the letter properly and are very interesting to children. Thompson et al. [27] stated that drawing letter shapes by tracing paths on screen improved writing capabilities of most students and is an essential educational activity of learning to write letters of the alphabet and this was followed in our program. Patchan and Puranik [28] also stated that writing on tablets are highly effective as it provides two types of feedback extrinsic and intrinsic; the extrinsic feedback is the immediate feedback given by the tablet or computer once an error is made. The intrinsic is the one naturally occurring sensory information resulting from writing by one's finger over the visual feedback after seeing the result of what was written and that enhances writing skills

The items of the Dysgraphia Disability Scale test that showed non-significant result after training as proprioceptive function except for graphethesia as the other functions were already good in most of the patients before therapy.

Improvement in the fine motor function and the perceptual motor function subtests was due to activities like coloring with the brush, using scissors, playing with playdough, and tracing through transparent paper all aimed at improving fine motor abilities of the children and that lead to improving the tested items.

The program was conducted on children aged from 6.5 to 10 years as this is the suitable age when the mature grip is achieved and it is very important to model writing in this young age to prevent further worsening of the handwriting. Training gave better results with elder age group and this goes with Overvelde and Hulstijn [29] study that stated that the percentage of children who were classified as dysgraphic decreased strongly from 37 to $17 \%$ in grade $2(7,8)$ years and diminished further to a low and stable rate of $6 \%$ in grade
$3(8,9)$ years. Stability in handwriting quality occur during this age (8-9 years); that is why the chosen age is the golden age to work on dysgraphia before handwriting stability.

Occupational therapy was applied parallel with dysgraphia training in the motor dysgraphic child, with teachers of special education and physiotherapy at a private clinic.

The child with spatial dysgraphia was given activities and accommodation techniques to be done at home and school in concomitant with dysgraphia therapy sessions.

Dyslexic dysgraphic children were receiving dyslexia therapy sessions in concomitant with dysgraphia therapy sessions at the phoniatric unit as they gain most benefits when the two programs were applied together as advised by Kohnen et al. [30].

After finishing the therapy program, the children were asked to complete their dysgraphia training for more than the 6-month duration (1 year) in a trial to increase the speed of writing without losing the legibility and alignment. Until reaching this point, they were advised to get an official report from the phoniatric unit to the Ministry of Education to get the following during exams:

- Extra time

- \pm Multiple choice questions

- \pm A teacher to read the questions and write the answers for the child

\section{Conclusion}

The Arabic dysgraphia training program is an effective multisensory approach tool in the training of different types of dysgraphia.

\section{Recommendations}

Applying the program on a wide scale all over the Egyptian governates.

\section{Supplementary information}

Supplementary information accompanies this paper at https://doi.org/10. 1186/s43163-020-00041-1.

\section{Additional file 1: Appendix}

\section{Acknowledgements}

Not applicable.

\section{Consent for the publication}

Written informed consent for publication of the patient's details was obtained from the patient.

Authors' contributions

Prof. S E and Prof. N R have an equal role in design. Assistant Prof. M D and lecturer of phoniatrics Dr. R S have an equal role in design, work, statistical analysis, and manuscript writing. All authors have read and approved the manuscript for publication. 


\section{Funding}

This research received no specific grant from any funding agency in the public, commercial, or not-for-profit sectors.

\section{Availability of data and materials}

This is available. All data generated or analyzed during this study are included in this published article and its supplementary information files.

\section{Competing interest}

The authors declare that they have no competing interests.

\section{Ethics approval and consent to participate}

A quasi-experimental study was carried out following the approval of the Research Ethical Committee of Faculty of Medicine, Phoniatric Unit, Ain Shams University (no. 158212013), on twenty consecutive cases (14 males and 6 females), ranging in age between 6.5 and 10 years, complaining of learning disability having dysgraphia with or without dyslexia, with $I Q \geq 90$. All patients' parent or legal guardians were informed about the aim of the study, and the procedure was fully explained to them. Written informed consent was taken from each child's parents that they know the type of evaluation and training and the duration of the given training program given to their child.

\section{Author details}

'Unit of Phoniatrics, ENT Department, Faculty of Medicine, Tanta University, Tanta, Egypt. ${ }^{2}$ Faculty of Medicine, Ain Shams University, Cairo, Egypt.

Received: 15 April 2020 Accepted: 24 September 2020

Published online: 28 October 2020

\section{References}

1. Felder-Puig R, Baumgarther M, Topf R (2008) Health-related quality of life in Austrian elementary school children, Medical Care:46

2. Rosenblum S, Livneh-Zirinski M (2008) Handwriting process and product characteristics of children diagnosed with developmental coordination disorder. Human Movement Science 27:200-214

3. James $\mathrm{KH}$. Sensori-motor experience leads to changes in visual processing in the developing brain. Developmental Science. 2010;13:279-88.

4. Schwellnus, H. , Carnahan, H. , Kushki, A. , Polatajko, H. , Missiuna, C. , Chau, T. (2013). Writing forces associated with four pencil grasp patterns in grade 4 children. The American Journal of Occupational TherapyMarch/April 2013, Volume 67, Number 2

5. Mirdehghan M (2010) Persian, Urdu, and Pashto: a comparative orthographic analysis. Writing Systems Research 2(1):9-23

6. Ahmad, Syed Barakat. Introduction to Qur'anic script. ISBN 978-1-136-11138-9. OCLC 1124340016

7. Stefan Widany (2011). The history of Arabic calligraphy: an essay on its greatest artists and its development. GRIN Verlag. ISBN 978-3-640-93875-9.)

8. Deuel RK (1995) Developmental dysgraphia and motor skill disorders. Journal of Child Neurology 10(1):6-8

9. Berninger WW, Wolf BJ (2009) Teaching students with dyslexia and dysgraphia: lessons from teaching and science. Paul H, Baltimore, Maryland

10. Nicolson RI, Fawcett AJ (2011) Dyslexia, dysgraphia, procedural learning and the cerebellum. Cortex. 47(1):117-127

11. Melika L.(1998): Standford Binnet Intelligence Scale (4th Arabic version). Second edition. Victor Kirolos Publishing, Cairo.

12. El-Sady S, El-Shoubary A, El-Assal N, Abou-Elsaad T, Afsah O (2011) The efficacy of a language-based training program as a phoniatric approach for remediation of Egyptian learning-disabled children. Unpublished thesis for partial fulfillment of doctoral degree presented by Afsah O, faculty of medicine. In: Ain Shams University

13. El-Sady S., El-Shoubary A., Abd El-Azziz N., Azzam A. (1996): Illinois test of psycholinguistic abilities. Unpublished M.Sc. Essay of Phoniatrics presented by A. Azzam, faculty of medicine, Ain Shams university.

14. Aboras Y, Abdou R, Kozou H (2008) Development of Arabic test for assessment of dyslexia in Egyptian children. Bulletin of Alexandria Faculty of medicine 44(3):653-662

15. Gamal N. and Gouda (2011): Dysgraphia Dysability Scale (DDS), ESPL 2011. ****

16. Amundson, S.J. (2001): Prewriting and handwriting. In: J. Case-Smith (Ed.). Occupational therapy for children (4th edition, pp. 545-570). St. Louis, MO: Mosby.
17. Bundy, A. C. \& Murray, E. A. (2002): Sensory integration: A. Jean Ayres' theory revisited. In. A. C. Bundy, S. J. Lane, \& E. A. Murray (Eds), Sensory integration: theory and practice (2nd ed., pp. 3-33). Philadelphia: F. A. Davis.

18. Malburg \& Coventry (2012). The Montessori curriculum: preschool handwriting activities. http://www.brighthubeducation.com/teachingpreschool/44160-montessori-handwriting-activities/

19. Chandar Kumar N (June 2015) (2013). A single session of NCK therapy in ameliorating excessive pressure while writing in a child with writing difficulty. International. Journal of Science and Research (IJSR) ISSN. Volume 4 Issue:6 www.ijsr.net

20. Winston and Professor R (2003) The Human Mind. Where does our personality come from? Can we explain love at first sight? What do me mean by 'intelligence' or 'genius'? Can science explain intuition or deja-vu? Focusing on our day-to-day experiences. In: BBC1

21. Smith, B.(2008). Why is handwriting so challenging. http://www. barbarasmithoccupationaltherapist.com/whyishandwritingsochallenging.html

22. Lippincott, C. (2016) .Ideas for teaching handwriting to children with dysgraphia. http://www.decodingdyslexiaor.org/wp-content/uploads/2 014/11/ldeas-for-Teaching-Handwriting-to-a-Child-with-Dyspraphia.pdf

23. Case Smith J (2002) Effectiveness of school-based occupational therapy intervention on handwriting. The American Journal of Occupational Therapy 56(1):17-25

24. Heerlen, (2013). The Cognitive Orientation to daily Occupational Performance (CO-OP) approach, CO-OP academy meeting Toronto.

25. Jordan G (2016) Bsc, Michaud, F. Effectiveness of an intensive handwriting program for first grade students, Kaiser, $M$

26. Mamaa Y, Ichtb M (2014) Auditioning the distinctiveness account: expanding the production effect to the auditory modality reveals the superiority of writing over vocalizing Published online: 06 Dec 2014

27. Thompson,R. , Tanimoto,S., Berninger,V. (2016). Interaction in writing instruction on tablets. Springer International Publishing Switzerland 2016 T. Hammond et al. (eds.), Revolutionizing education with digital ink, human-computer interaction series, DOI https://doi.org/10.1007/ 978-3-319-31193-7_4

28. Patchan and Puranik (2016) Computers \& education. Computers and Eduction Elsevier journal vol. 102 pages 1-258 nov. 2016

29. Overvelde A, Hulstijn W (2011) Handwriting development in grade 2 and grade 3 primary school children with normal, at risk, or dysgraphic characteristics. Research in Developmental Disabilities 32:540-548. http://dx. doi.org/. https://doi.org/10.1016/j.ridd.2010.12.027

30. Kohnen, S., Nickels, L., \& Coltherat, M. (2010). Skill generalization in teaching spelling to children with learning difficulties. Australian Journal of Learning Difficulties. Volume 15, - Issue 2

\section{Publisher's Note}

Springer Nature remains neutral with regard to jurisdictional claims in published maps and institutional affiliations.

\section{Submit your manuscript to a SpringerOpen ${ }^{\circ}$ journal and benefit from:}

- Convenient online submission

- Rigorous peer review

- Open access: articles freely available online

- High visibility within the field

- Retaining the copyright to your article

Submit your next manuscript at $>$ springeropen.com 